\title{
ON ORDERING AND DANGER OF CIAIM FREQUENCY DISTRIBUTIONS
}

\author{
M. J. Goovaerts*
}

The notion of ordering and danger of claim size distributions is extended to claim frequency cistributions.

\section{INTRODUCTION}

Let us consider the Swiss premium calculation principle. For any given risk the premium $\pi(X, v, z)$ is defined as the root of the equation in $p$ :

$$
E(v(S-z p))=v((1-z) p)
$$

where $v$ denotes a twice differentiable function with $v^{\prime}(t)>0, v^{\prime \prime}(t) \geqslant 0$, for $-\infty<t<+\infty$. Let $\pi(X, \alpha, v, z)$ denote the stop-loss premium for retention limit $\alpha$, then it is the root of the equation in $p$ :

$$
v((1-z) p)=v(-z p) F_{S}(\alpha)+\int_{\alpha}^{\infty} v(t-\alpha-z p) d F_{S}(t)
$$

We will recall here a definition of a partial ordering among risks introduced in Bühlmann, Gagliardi, Gerber and Straub (1977).

\section{Definition 1}

Let $G, H$ be any distributions on the real line, then we say that $G<H$ if

i) $\int_{0}^{\infty} x d G(x)<\infty$

ii) $\int_{i}^{\infty}(x-t) d G(x) \leqslant \int_{1}^{\infty}(x-t) d H(x) \quad-\infty<t<+\infty$

We also recall the following definition of dangerous distributions.

\section{Definition 2}

A distribution $H$ is called more dangerous than a distribution $G$ if the first moments say $\mu_{G}$ and $\mu_{I}$ exist and $\mu_{G} \leqslant \mu_{H}$ and if there is a constant $\beta$ such that

$$
\begin{aligned}
& G(x) \leqslant H(x) \quad x<\beta \\
& G(x) \geqslant H(x) \quad x \geqslant \beta
\end{aligned}
$$

* 15.U. Leuven, Jekenstraat 2, 13-300o Ieuven. The note and especially the proof of lemma 3 has benefitted from a remark by Dr. G. Taylor. It has also benefitted from a discussion with Prof. H. Bülılmann and Prof. H. Gerber. 
In BÜILLMANN ET AL (1977) the ordering of risks as well as the notion of dangerousness has been introduced for claim size distributions. The aim of the present contribution consists in gencralizing these notions for claim frequency distributions. As has been suggested by Prof. $\mathrm{H}$. Bühlmann the same definitions can be adapted for claim frequency distributions.

\section{SOME DISCRETE INEQUALITIES}

We will formulate two lemmas on discrete inequalities involving convex functions. More general versions of these inequalities can be found in KARLIN and STUDDEN (1966).

\section{Lemma 1}

Let $\varphi$ be a convex function, let $\left\{a_{v} ; v=0, \ldots, n\right\}$ be a set of real numbers. Then

$$
\sum_{v=0}^{n} \varphi(v) a_{v} \geqslant 0 \quad \text { for } \forall \text { convex } \varphi
$$

if and only if

$$
\sum_{v-0}^{n}(u-k)+a_{v} \geqslant 0 \quad k=0, \ldots, n
$$

and

$$
\sum_{\nu=0}^{n} a_{\nu}=0
$$

Proof: See Karlin and Studden (1966).

\section{Lemma 2}

Let $\left\{a_{v}: v=0, \ldots, n\right\}$ be a set of real numbers, such that

$$
\begin{aligned}
& \sum_{u=0}^{n} v a_{0} \leqslant 0, \sum_{0=0}^{n} a_{v}=0 \\
& \sum_{v=0}^{k} a_{0} \leqslant 0 \quad k=0, \ldots, x
\end{aligned}
$$$$
\sum_{v=0}^{k} a_{0} \geqslant 0 \quad k=x+1, \ldots, n
$$

then

$$
\sum_{u=0}^{n}(u-r)+a_{u} \leqslant 0 \quad r=0,1, \ldots, n
$$

Proof: Sce Karlin and Studden (1966). 
Next we will formulate and prove the following:

Lemma 3 .

$$
y(n)=\int_{\alpha}^{\infty}(v(x-\alpha-z p)-v(-z p)) d F^{* n}(x)
$$

with $v^{\prime}>0, v^{\prime \prime} \geqslant 0$, denotes a convex sequence.

Proof: We will aim our attention first to the proof of the convexity of the special sequence.

$$
y_{0}(n)=\int_{\alpha}^{\infty}((x-\alpha-z p)-(-z p)) d F^{* n}(x) .
$$

We thus have to prove that

$$
y_{0}(n+2)-2 y_{0}(n+1)+y_{0}(n) \geqslant 0
$$

which is of course equivalent with

$$
y_{0}(n+1) \leqslant \frac{1}{2} y_{0}(n)+\frac{1}{2} y_{0}(n+2) .
$$

Firstly we limit ourselves to the special case $n=0$. We then have to show

$$
\int_{\alpha}^{\infty}(x-\alpha) d F(x) \leqslant \frac{1}{2} \int_{\alpha}^{\infty}(x-\alpha) d F^{0}(x)+\frac{1}{2} \int_{\alpha}^{\infty}(x-\alpha) d F^{* 2}(x) .
$$

This incquality is a special case of the more general result obtained in BüHLMANN ET AL (1977)

$$
\sum_{i=1}^{2} E\left(\left(X_{i}-\alpha\right)_{+}\right) \leqslant E\left(\left(\sum_{i=1}^{2} X_{i}-\alpha\right)_{+}\right)
$$

where $X_{1}$ and $X_{2}$ are distributed according to $F$ with $F(0)=0$.

Next we prove that the inequality (7) implies the following:

$$
y_{0}(n+3)-2 y_{0}(n+2)+y_{0}(n+1) \geqslant 0
$$

For the present choice of $v(x)=x$ one obtains

$$
y_{0}(n)=\int_{\alpha}^{\infty}\left(1-F^{*} n(x)\right) d x
$$

Consequently:

$$
\begin{aligned}
y_{0}(n+2) & =\int_{\alpha}^{\infty}\left(1-F^{*(n+2)}(x)\right) d x \\
& =\int_{\alpha}^{\infty}(1-F(x)) d x+\left(\int_{\alpha}^{\infty} F(x)-F^{*(n+2)}(x)\right) d x .
\end{aligned}
$$


Write $E(\alpha)$ for the first term on the r.h.s., then:

$$
\begin{aligned}
y_{0}(n+2) & =E(\alpha)+\int_{\alpha}^{\infty}\left\{F(x)-\int_{0}^{x} F^{*(n+1)}(x-y) d F(y)\right\} d x \\
& =E(\alpha)+\int_{\alpha}^{\infty} \int_{0}^{x}\left(1-F^{*(n+1)}(x-y)\right) d F(y) d x .
\end{aligned}
$$

Reversal of the order of integration gives:

(9) $\quad y_{0}(n+2)=E(\alpha)+\left\{\int_{0}^{\alpha} \int_{\alpha}^{\infty}+\int_{\alpha}^{\infty} \int_{y}^{\infty}\right\}\left(1-F^{*(n+1)}(x-y)\right) d x d F(y)$.

But because ( 7 ) holds one still has the following inequality

$$
\int_{\beta}^{\infty}\left(1-F^{*(n+2)}(x-y)\right) d x \leqslant \frac{1}{2} \int_{\beta}^{\infty}\left(1-F^{*(n+2)}(x-y)\right) d x+\frac{1}{2} \int_{\beta}^{\infty}\left(1-F^{* n}(x-y)\right) d x .
$$

Inserting the r.h.s. into the r.h.s. of (9) gives:

$$
\begin{aligned}
y_{0}(n+2) \leqslant E(\alpha) & +\frac{1}{2}\left\{\int_{0}^{\alpha} \int_{\alpha}^{\infty}+\int_{\alpha}^{\infty} \int_{y}^{\infty}\right\}\left(1-F^{*(n+2)}(x-y)\right) d x d F(y) \\
& +\frac{1}{2}\left\{\int_{0}^{\alpha} \int_{\alpha}^{\infty}+\int_{\alpha}^{\infty} \int_{\nu}^{\infty}\right\}\left(1-F^{* n}(x-y)\right) d x d F(y) \\
& =\frac{1}{2} y_{0}(n+3)+\frac{1}{2} y_{0}(n+1) .
\end{aligned}
$$

Hence, our lemma has been shown to hold in case $y(n)=y_{0}(n)$. Next we will show that the convexity of $y_{0}(n)$ implies this of $y(n)$. By means of two successive partial integrations one is faced with the following equality:

$$
\begin{aligned}
\int_{\alpha}^{\infty}(v(x-\alpha-z p) & -v(-z p)) d F^{* n}(x)=v^{\prime}(-z p) \int_{\alpha}^{\infty}\left(1-F^{* n}(x)\right) d x \\
& +\int_{\alpha}^{\infty} v^{\prime \prime}(t-\alpha-z p) \int_{\alpha}^{\infty}\left(1-F^{* n}(x)\right) d x .
\end{aligned}
$$

But

$$
\int_{\beta}^{\infty}\left(1-F^{* n}(x)\right) d x \leqslant \frac{1}{2} \int_{\beta}^{\infty}\left(1-F^{*(n-1)}(x)\right) d x+\frac{1}{2} \int_{\beta}^{\infty}\left(1-F^{*(n+1)}(x)\right) d x .
$$

Hence inserting the second member of the inequality in (10) gives:

$$
y(n)-2 y(n-1)+y(n-2) \geqslant 0 \quad \text { q.e.d. }
$$

3. CONCLUSIONS

We are now in the position to introduce the notion of dangerousness and ordering of claim frequencies in analogy to the corresponding definitions for claim size distributions. 


\section{Definition 3}

Let $G, H$ be two claim frequency distributions, then $G<H$ if

$$
\begin{aligned}
\text { i) } & \int_{0}^{\infty} x d G(x)<\infty \\
\text { ii) } \quad & \int_{i}^{\infty}(x-t) d G(x) \leqslant \int^{\infty}(x-t) d H(x) 0<t<\infty
\end{aligned}
$$

\section{Definition 4}

A claim frcquency distribution $H$ is called more dangerous than a claim frequency distribution $G$ if the expected claim numbers $\mu_{G}$ and $\mu_{I I}$ exist and if there is a constant $\beta$ such that

$$
\begin{aligned}
& G(x) \leqslant H(x) \quad x<\beta \\
& G(x) \geqslant H(x) \quad x \geqslant \beta
\end{aligned}
$$

\section{Theorem 1}

If a claim frequency distribution $H$ is more dangerous than a claim frequency distribution $G$ then $G<H$.

Proof: Immediately from lemma 2.

\section{Theorem 2}

$$
\text { Let } \begin{aligned}
F_{S_{1}}(x) & =\sum_{n=0}^{\infty} p_{n} F^{* n}(x) \\
F_{S_{2}}(x) & =\sum_{n=0}^{\infty} p_{n}^{\prime} F^{* n}(x) \\
G(x) & =\sum_{n=0}^{[x]} p_{n}, H(x)=\sum_{n=0}^{[x]} p_{n}^{\prime}
\end{aligned}
$$

If $G<H$ then $F_{S_{1}}<F_{S_{2}}$.

Proof: immediately from lemma 1 and lemma 3.

RETERENCES

Bünlmann, H., Gagliardi, B., Gerbler, H. U. and Straub, E. (1977). Some Inequalities for Stop-Loss Premiums, Astin Bullatin 9, 75-83.

KArLIn, S. and Stunden, W. (Eds.) (1966). Tchebychaff Systems: With Applications in Analysis and Statistics. Interscience l'ublishers, New York. 